\title{
Profil biochimique et hématologique des patients drépanocytaires homozygotes en phase stationnaire au centre National de Transfusion Sanguine de Dakar
}

\author{
Dominique DOUPA ${ }^{1 *}$, Moustapha DJITE ${ }^{2}$, Pape Madieye GUEYE ${ }^{2}$, Moussa SECK ${ }^{3}$, \\ Blaise Felix FAYE ${ }^{3}$, Sidy Mohamed $\mathrm{SECK}^{4}$, Fatou DIALLO ${ }^{5}$, Arame NDIAYE ${ }^{5}$, \\ Abdourahmane SAMBA $^{5}$, Fatou CISSE ${ }^{5}$, Alassane DIATTA ${ }^{5}$, Ibrahima DIAGNE ${ }^{6}$ \\ et Saliou DIOP ${ }^{3}$
}

\author{
${ }^{1}$ Laboratoire de Biochimie UFR Sciences de la Santé, UGB, Saint-Louis, Sénégal. \\ ${ }^{2}$ Laboratoire Biochimie Pharmaceutique, FMPO, UCAD, Dakar, Sénégal. \\ ${ }^{3}$ Service Hématologie, FMPO, UCAD, Dakar, Sénégal. \\ ${ }^{4}$ Service de Néphrologie UFR Sciences de la Santé, UGB, Saint-Louis, Sénégal. \\ ${ }^{5}$ Laboratoire de Biochimie Médicale, FMPO, UCAD, Dakar, Sénégal. \\ ${ }^{6}$ Service de Pédiatrie UFR Sciences de la Santé, UGB, Saint-Louis, Sénégal. \\ *Auteur correspondant; E-mail :d_doupa@hotmail.com; Tél :0022177/649/05/62
}

\section{RESUME}

La drépanocytose est caractérisée par une grande variabilité d'expression clinique et biologique qui dépend de plusieurs facteurs. L'objectif de ce travail était d'étudier les perturbations biologiques des patients drépanocytaires homozygotes en phase stationnaire. Il s'agit d'une étude prospective transversale ayant inclus 100 drépanocytaires homozygotes en phase stationnaire d'âge supérieur à 15 ans. Chaque patient a bénéficié de deux tubes de prélèvements pour l'étude des paramètres hématologiques et pour les dosages biochimiques. Un questionnaire a été utilisé pour recueillir les variables épidémiologiques, cliniques et biologiques. Ces données ont été saisies et analysées par le logiciel Epi-info 7.2. Une valeur $\mathrm{p} \leq 0,05$ a été considérée comme significative. L'âge moyen des patients était de $(28 \pm 8,94)$ ans, les extrêmes $(15-57$ ans) et le sex-ratio $(\mathrm{H} / \mathrm{F})$ de 0,75 . Le taux d'hémoglobine de base était en moyenne de $(8,2 \pm 1,4 \mathrm{~g} / \mathrm{dl})$, les globules blancs de $(12352 \pm$ $\left.6.906 / \mathrm{mm}^{3}\right)$, les plaquettes $\left(439.920 \pm 139.000 / \mathrm{mm}^{3}\right)$, la TCMH $(31,18 \mathrm{pg} \pm 4,12 \mathrm{pg})$. Le taux d'hémoglobine S était en moyenne de $(87,79 \pm 8,19 \%)$ et l'hémoglobine F $(\mathrm{HbF})(9,5 \pm 8,3 \%)$. Le taux HbF était associée de façon significative avec le syndrome thoracique aigu et la lithiase biliaire $(\mathrm{r}=0,56 ; \mathrm{p}=0,003)$. Cette étude a montré que les perturbations biologiques sont fréquentes chez les dans la drépanocytaires homozygotes. Leur connaissance servira de base de comparaison lors des crises et pour évaluer l'efficacité du traitement. (C) 2017 International Formulae Group. All rights reserved.

Mots clés : Drépanocytose SS, phase stationnaire, perturbations biochimiques, hématologiques. 


\title{
Biochemical and hematological profile of homozygous sickle cell patients in stationary phase at the Blood Transfusion National Center of Dakar
}

\begin{abstract}
Sickle cell disease is frequently associated with a high clinical and biological variability. This study aimed to describe biological abnormalities in Senegalese patients with homozygous sickle cell disease. In a cross-sectional study, we included 100 patients aged 15 years with homozygous sickle cell disease. Hematological and biochemical parameters were assessed for each patient. Clinical, epidemiological and biological variables were collected using a questionnaire. Data were analyzed using Epi-info 7.2. The mean age of patients was $(28 \pm 8.94)$ years and sex ratio $(\mathrm{H} / \mathrm{F})$ was 0,75 . The basal hemoglobin level was $(8.2 \pm 1.48$ $\mathrm{g} / \mathrm{dl})$, white blood cells $\left(12352 \pm 6.906 / \mathrm{mm}^{3}\right)$, platelets $\left(439.920 \pm 139.000 / \mathrm{mm}^{3}\right)$, TCMH $(31,18 \pm 4.12 \mathrm{pg})$. The mean Hemoglobin $\mathrm{S}$ was $(87.79 \pm 8.19 \%)$ and hemoglobin $\mathrm{F}(\mathrm{HbF})(9.5 \pm 8.3 \%)$. HbF was associated with acute chest syndrome and biliary lithiasis $(\mathrm{r}=0,56 ; \mathrm{p}=0,003)$. This study showed that biological abnormalities in homozygous sickle cell patients in the stationary phase are frequent. The base line characteristics will help for comparison during next crises and for assess effectiveness of treatment.

(C) 2017 International Formulae Group. All rights reserved.
\end{abstract}

Keywords: Sickle cell disease, stationary phase, biochemical disturbances, hematological.

\section{INTRODUCTION}

La drépanocytose concerne cinquante millions de personnes à travers le monde ce qui en fait la maladie génétique la plus répandue (Sergent et al., 2013). Bien qu'on la rencontre dans le monde entier, on la rencontre principalement chez les sujets de race noire, originaires d'Afrique équatoriale ou d'Afrique subsaharienne. Il est estimé chaque année que plus de 230000 enfants drépanocytaires naissent en Afrique Subsaharienne contre 2600 en Amérique du Nord et 13000 en Europe (Serjeant 2013; Bardakjian et al., 2000). Au Sénégal, sa prévalence est estimée à $10 \%$ dont $1 \%$ pour les formes homozygotes (Diagne et al., 2000). Chez ces patients, une grande variabilité des données biologiques ont été observées selon le génotype, le sexe, l'âge des patients avec des différences selon que l'examen ait été réalisé en phase stationnaire au cours d'une crise ou complication (Marie Redelsperger et al., 2003 ; Girot et al., 2004). Il existe en outre très peu d'études locales sur le statut biologique des patients drépanocytaires homozygotes en phase stationnaire. La phase stationnaire étant définie par l'absence de complication et de manifestation aigue de la drépanocytose dans les 15 jours précédents le recrutement. C'est pour cette raison que nous sommes fixés comme objectifs: (a) de déterminer les perturbations biochimiques et hématologiques au cours de la drépanocytose homozygote en phase stationnaire; (b) de les utiliser comme de base de comparaison lors des crises pour mieux évaluer l'efficacité du traitement.

\section{MATERIEL ET METHODES}

Une étude prospective, descriptive et transversale a été menée dans les services d'hématologie clinique du Centre National de Transfusion Sanguine (CNTS) et le service de Biochimie de l'hôpital National Universitaire de Fann sur une période de six mois (Septembre 2015 à Février 2016). Elle a concerné les patients drépanocytaires homozygotes en phase stationnaire âgés de 15 ans et plus régulièrement suivis dans ce centre. Chaque patient a bénéficié de deux tubes de prélèvements sur tube EDTA pour l'étude des paramètres hématologiques et sur 
tube sec pour les dosages biochimiques. Il s'agissait d'un échantillonnage systématique aléatoire simple dans une population de 1400 drépanocytaires homozygotes SS suivis dans ce centre.

\section{Critères d'inclusion}

Les critères de sélection des patients étaient :

- Etre âgé de 15 ans et plus

- Accord de participation à l'étude par consentement écrit, éclairé et signé du sujet

- Absence de manifestation ou de complication aigue de la drépanocytose dans les 15 jours précédant le recrutement

- Profil drépanocytaire homozygote confirmé par l'électrophorèse de l'hémoglobine.

\section{Critères de non inclusion}

Les critères de non inclusion étaient :

- Suivi irrégulier (moins de 3 consultations) durant l'année précédant le recrutement

- Manifestation ou complication aigue de la drépanocytose observée dans les 15 jours précédant le recrutement

- Autres hémoglobinopathies

- Refus volontaire de participer à l'étude.

\section{Critères d'exclusion}

Les patients présentant d'autres maladies chroniques ont été exclus de cette étude ainsi que les patients dont les dossiers médicaux sont incomplets.

\section{Considérations éthiques}

Un consentement libre et éclairé (verbal ou écrit) de toutes les personnes impliquées dans cette étude a été obtenu au préalable.

\section{Paramètres hématologiques}

Chaque patient a bénéficié d'un prélèvement sanguin de cinq millilitres de sang total recueilli sur tube EDTA après ponction veineuse pour :

- la numération formule sanguine à l'aide du Coulter ABX pentra

- l'électrophorèse de l'hémoglobine par la technique capillaire grâce au Minicap Flex piercing de SEBIA sur du sang frais prélevé sur tube EDTA ou conservé à $2-7^{\circ} \mathrm{C}$ moins de sept jours. Les normes utilisées sont basées sur les valeurs de référence internationale pour l'hémogramme. Nous avons retenu les critères de décision suivants :

- l'anémie était définie par un taux d'hémoglobine $(\mathrm{Hb})<11 \mathrm{~g} / \mathrm{dl}$ et typée, selon le volume globulaire moyen (VGM), en anémie macrocytaire (VGM > $95 \mathrm{fl}$ ), normocytaire (80 $\mathrm{fl} \leq \mathrm{VGM} \leq 90 \mathrm{fl}$ ) et microcytaire (VGM $<80$ fl. Elle est considérée comme sévère si $\mathrm{Hb}$ était $<7 \mathrm{~g} / \mathrm{dl}$, modérée si $7 \mathrm{~g} / \mathrm{dl} \leq \mathrm{Hb}<9 \mathrm{~g} / \mathrm{dl}$ et légère si $9 \mathrm{~g} / \mathrm{dl} \leq \mathrm{Hb}<11 \mathrm{~g} / \mathrm{dl}$. Selon la teneur corpusculaire moyenne en hémoglobine(TCMH) dont la valeur varie de 27 à $31 \mathrm{pg}$, on distingue les anémies hypochromes $(\mathrm{TCMH}<27 \mathrm{pg}$ ), et les anémies normochromes $(27<\mathrm{TCMH}<31 \mathrm{pg})$.

- le taux normal des plaquettes est compris entre 200000 et $450000 / \mathrm{mm}^{3}$

- le taux normal des globules blancs 4000 à $10000 / \mathrm{mm}^{3}$

\section{Paramètres biochimiques}

Chaque patient a bénéficié de cinq millilitres de sang total sur tube sec, centrifugé à 3000 tours $/ \mathrm{mn}$ pendant cinq minutes. Les sérums ont été recueillis et conservés à moins quatre degré Celsius lorsque les dosages biochimiques ont été différés. Les paramètres biochimiques ont été dosés par méthode chimioluminescente grâce à l'automate multiparamétrique Cobas Roche. Les normes utilisées sont par conséquent celles en vigueur dans ce laboratoire et qui se rapprochent d'ailleurs de celles du consensus international: ASAT $<45 \mathrm{UI} / 1, \quad$ ALAT $<$ 40UI/1, CRP $<6 \mathrm{mg} / 1$. 


\section{Collecte des données et analyse statistique}

Il s'agit d'une étude transversale sur dossiers des patients avec l'établissement d'une fiche d'enquête. Pour chaque patient, les données épidémiologiques, cliniques, biochimiques et hématologiques ont été recueillies sur fiche d'enquête puis saisies sur ordinateur et analysées grâce au logiciel Epiinfo 7.0 du CDC, Atlanta, USA. Le test-t de STUDENT a été utilisé pour la comparaison des moyennes des variables quantitatives et le test de chi-2 pour les variables qualitatives. Le seuil de significativité pour les tests statistiques a été fixé à un $\mathrm{p}<0,05$.

\section{RESULTATS}

\section{Sur le plan épidémiologique}

Sur une période de six mois 100 patients drépanocytaires homozygotes ont été étudiés. La répartition des malades par âge et par sexe a fait apparaitre une légère prédominance féminine (57 femmes) soit $57 \%$, alors que la fréquence masculine avec 43 cas est de $43 \%$. L'âge moyen des patients a été de 28 ans avec un écart type de 8,3 (extrêmes 15 et 57 ans). La couche la plus importante était celle dont l'âge est comprise entre 15 et 25 ans (52 cas soit $52 \%$ de la population). On remarque que le pourcentage des patients drépanocytaires a diminué avec l'âge. Les patients ayant un âge supérieur à 46 ans étaient au nombre de 6 soit $6 \%$ de la population. La Figure 1 illustre la répartition des patients en fonction du sexe. Il en ressort que la majorité de nos patients étaient originaires de Dakar (55 patients) soit $55 \%$ de la population.

\section{Sur le plan clinique}

Les complications cliniques étaient dominées par des crises vaso-occlusives 47 patients soit $(47 \%)$ de la population étudiée dont 38 ont fait 2 crises. Le Tableau 1 résume ces complications.

\section{Sur le plan hématologique}

Le taux d'hémoglobine de base était compris entre 5,2 et $12 \mathrm{~g} / \mathrm{dl}$ avec une moyenne de $8,2 \pm 1,4 \mathrm{~g} / \mathrm{dl}$. La concentration corpusculaire moyenne en hémoglobine (CCMH), la teneur corpusculaire moyenne en hémoglobine (TCMH) étaient respectivement de $35,28 \pm 3,46 \%$ et $31,18 \pm 4,1 \mathrm{pg}$. Une anémie a été observée chez $94 \%$ de patients. Le degré d'anémie est très variable, $18 \%$ présentait une anémie sévère, $48 \%$ une anémie modérée et $34 \%$ une anémie mineure. La Figure 2 illustre cette répartition.

Pour les leucocytes, la moyenne était de $12345 \pm 6900 / \mathrm{mm}^{3}$ avec des extrêmes allant 3500 à $49000 / \mathrm{mm}^{3}$. L'hyperleucocytose a été observée chez $53 \%$ des patients. Le taux moyen des plaquettes était de $439000 \pm 139.330 / \mathrm{mm}^{3}$ avec des extrêmes allant de 180000 à $828000 / \mathrm{mm}^{3}$. 47\% des patients présentaient une thrombocytose. Le Tableau 2 rapporte les valeurs moyennes des paramètres hématologiques des malades.

\section{Sur le plan Biochimique}

Les moyennes des fractions hémoglobiniques étaient respectivement de $87,78 \pm 8,19 \%$ pour l'hémoglobine $\mathrm{S}, 2,72 \pm$ $1,04 \%$ pour l'hémoglobine A2 et $9,50 \pm$ $8,34 \%$ pour l'hémoglobine $\mathrm{F}$. Le taux d'hémoglobine $F$ était corrélé de façon statistiquement significative avec le syndrome thoracique aigue (STA) et la lithiase biliaire ( $\mathrm{r}$ $=0,56 ; \mathrm{p}<0,003)$. La protéine $\mathrm{C}$ réactive (CRP) était en moyenne de 12,87 $\pm 21,9$ $\mathrm{mg} / \mathrm{L}$. Elle était corrélée de façon statistiquement significative avec la leucocytose $(r=0,72 ; p<0,01)$. Les transaminases (ASAT et ALAT) étaient respectivement de 40,63 $\pm 32,9 \mathrm{UI} / \mathrm{L}$ et 28,62 $\pm 44,52 \mathrm{UI} / \mathrm{L}$. La majorité des patients avaient bilan hépatique normal dont ASAT chez 73\% et L'ALAT chez $82 \%$ des patients de notre série. Les résultats des variables biochimiques ont été consignés dans le Tableau 3. 


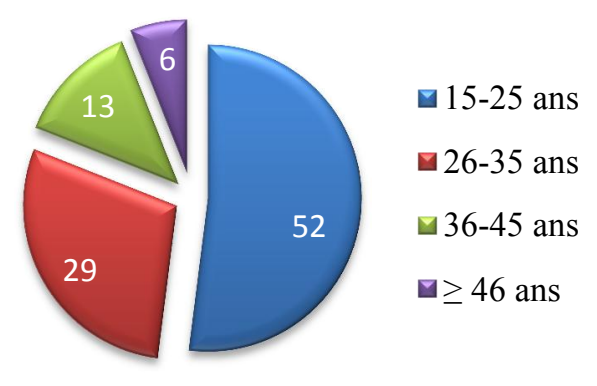

Figure 1: Répartition des patients selon l'âge.

Tableau 1 : Répartition des patients selon les complications observées.

\begin{tabular}{lcc}
\hline Complications cliniques & Fréquence absolue & Fréquence relative \\
\hline Crises vaso-occlusives & 47 & $47 \%$ \\
Anémies & 28 & $28 \%$ \\
Protéinuries & 17 & $17 \%$ \\
Lithiases biliaires & 09 & $09 \%$ \\
Pneumonies & 09 & $09 \%$ \\
Ostéonécrose (hanche/épaule) & 08 & $08 \%$ \\
Priapisme & 07 & $07 \%$ \\
Ulcère de jambe & 07 & $07 \%$ \\
Syndrome thoracique aigu & 06 & $06 \%$ \\
Accident vasculaire cérébral & 04 & $04 \%$ \\
Septicémie & 2 & $02 \%$ \\
Ostéomyélite & 1 & $01 \%$ \\
\hline
\end{tabular}

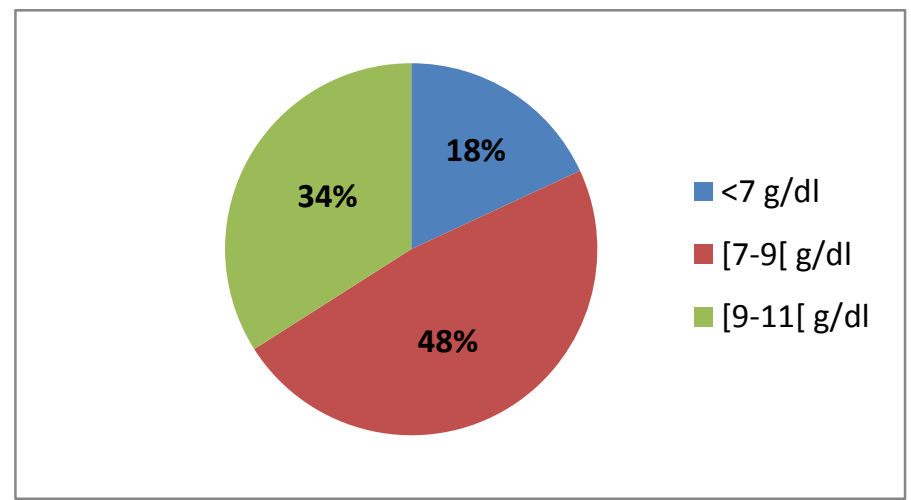

Figure 2 : Répartition des patients en fonction du degré d'anémie. 
Tableau 2 : Variations des paramètres hématologiques.

\begin{tabular}{lccc}
\hline $\begin{array}{l}\text { Paramètres } \\
\text { hématologiques }\end{array}$ & Moyennes \pm écart type & Valeurs extrêmes & $\begin{array}{c}\text { Valeurs } \\
\text { normales }\end{array}$ \\
\hline Hb de base (g/dl) & $8,2 \pm 1,4$ & $05-12$ & $12-16$ \\
Hb habituel (g/dl) & $8,5 \pm 1,5$ & $04,5-13,4$ & $12-16$ \\
VGM (fl) & $83,2 \pm 3,5$ & $75-96$ & $80-95$ \\
CCMH (\%) & $35,28 \pm 3,4$ & $25,9-45,5$ & $30-35$ \\
TCMH (pg) & $31,18 \pm 4,1$ & $21,5-41,4$ & $27-31$ \\
$\begin{array}{l}\text { Globules blancs } \\
\text { (unité/mm }\end{array}$ & $12352 \pm 6.900$ & $3590-49100$ & $4000-10000$ \\
Plaquettes (unité/mm & & $128000-828000$ & $200000-450000$ \\
\hline
\end{tabular}

Tableau 3 : Variations des paramètres biochimiques.

\begin{tabular}{lccc}
\hline Paramètres biochimiques & Moyenne \pm écart type & Valeurs extrêmes & $\begin{array}{c}\text { Valeurs } \\
\text { normales }\end{array}$ \\
\hline HbS (\%) & $87,79 \pm 8,2$ & $60,1-98,8$ & 0 \\
HBA2 (\%) & $2,7 \pm 1,04$ & $0,4-6,6$ & $1-3 \%$ \\
HB F (\%) & $9,5 \pm 8,3$ & $0-37,5$ & Traces \\
CRP (mg/l) & $12,9 \pm 21,9$ & $0,29-149,26$ & $0-6$ \\
ASAT (UI/l) & $40,63 \pm 32,9$ & $12,9-319,91$ & $10-40$ \\
ALAT (UI/l) & $28,62 \pm 44,52$ & $0,9-434,12$ & $10-45 \mathrm{UI} / \mathrm{L}$ \\
\hline
\end{tabular}

\section{DISCUSSION}

L'objectif de ce travail était de dégager des paramètres biologiques qui serviront de base de référence lors des crises et qui permettrons d'évaluer l'efficacité de la prise en charge des patients drépanocytaires homozygotes (SS).

\section{Sur le plan épidémiologique}

La population d'étude était jeune avec un âge moyen de 28 ans ce qui est en parfait accord avec les études de Nacoulma et al. (2006) au Mali dont $80 \%$ des patients avaient un âge inférieur à 15 ans ainsi que Dahmani et al. (2016) au Maroc. On remarque que le pourcentage des patients drépanocytaires diminue avec l'âge ceci peut être expliqué par la mortalité précoce des sujets. Selon certains auteurs Dapa et al. (2008), la létalité de la maladie avant l'âge de 5 ans atteint $50 \%$ en l'absence de recours sanitaire adapté. Cependant d'autres auteurs ont rapporté plusieurs drépanocytaires homozygotes parvenus à l'âge adulte. Begue et al. (2009) avancent 10 à $50 \%$ de survie à 20 ans selon les séries.

Les patients de cette série étaient répartis en 43 hommes et 57 femmes avec un sex ratio $(H / F)$ de 0,75 ce qui rejoint les études faites au CHU de Brazzaville (Dokékias et al., 2011) et au niveau de la république démocratique du Congo (Shongo et al., 2015) dans lesquelles ils ont objectivé une prédominance féminine avec respectivement un sex ratio $(\mathrm{H} / \mathrm{F})$ de 0,8 et 0,9 . Par contre, dans deux études faites à 
Dakar (Diagne et al., 2000) et au Centre National de Référence de la drépanocytose au Niger (Mounkaila, 2015), ils ont retrouvé une légère prédominance masculine avec respectivement des sex ratio $(\mathrm{H} / \mathrm{F})$ de 1,02 et 1,2 . D'autres auteurs ne constatent aucune prédominance entre les deux sexes, c'est le cas de (Than et al., 2008). Ces différences seraient en rapport avec les données démographiques de chaque pays car la transmission de la tare se fait indépendamment du sexe (Mabiala et al., 2005).

La majorité des patients étaient originaire de Dakar et ceci se justifie par le fait que le seul centre spécialisé dans la prise en charge des adultes s'y trouve. Cela soulève la nécessité de créer d'autres centres de prise en charge dans toutes les régions du pays.

\section{Sur le plan clinique}

Par leur fréquence, les crises douloureuses dominent la symptomatologie de la maladie drépanocytaire. On les désigne souvent sous le terme de crises vasoocclusives. Elles peuvent être spontanées (une fois sur deux environ) ou provoquées par un facteur comme l'infection, le froid, l'acidose, la fièvre, la déshydratation ou toute situation entrainant une hypoxémie. Dans cette étude, les crises vaso-occlusives dominaient le tableau clinique dans (48\%) des cas. Ceci rejoint les deux études faites à Rabat (Harak et al., 2010) et à Dakar (Diagne et al., 2000) ou les crises vaso-occlusives représentaient la première manifestation clinique en terme de fréquence avec respectivement $80 \%$ et $67 \%$. Le syndrome anémique représentait la deuxième manifestation clinique en termes de fréquence, ce qui rejoint celle d'une étude malienne qui avait retrouvé $(35,3 \%)$ des cas (Doumbia et al., 2009).

\section{Sur le plan hématologique}

La moyenne du taux d'hémoglobine dans cette étude était de $8,2 \pm 1,4$. Cette moyenne est comparable à celles trouvées par plusieurs auteurs notamment au Maroc (Dahmani et al., 2016) et au mali (6) avec respectivement $7,9 \mathrm{~g} / \mathrm{dl}$ et $7,8 \mathrm{~g} / \mathrm{dl}$. Dans cette étude, la majorité des patients étaient anémiés
(94\%) dont presque la moitié présentait une anémie modérée (48\%). Les anémies observées dans cette étude étaient principalement de type normochromes normocytaire $(73 \%)$ et hypochrome microcytaire (17\%). Ces données sont en concordance avec les données de la littérature qui donnent principalement une anémie normochrome normocytaire au cours de cette affection (Nacoulma et al., 2006 ; Dahmani et al., 2016).

Pour beaucoup d'auteurs, l'hyperleucocytose serait habituelle dans la drépanocytose homozygote (Nacoulma et al., 2006; Dahmani et al., 2016). En phase stationnaire et en dehors de toute infection, certains patients peuvent présenter 25000 à $35000 / \mathrm{mm}^{3}$ avec une moyenne de $12000 \pm$ $3000 / \mathrm{mm}^{3}$. Nacoulma et al. (2006) a retrouvé une moyenne de $12530 / \mathrm{mm}^{3}$ dans sa population d'étude avec des extrêmes allant de de 8600 à $27700 / \mathrm{mm}^{3}$. Ces résultats concordent avec ces chiffres, en effet nous avons retrouvé une moyenne de $12345 \pm$ $6900 / \mathrm{mm}^{3}$

L'hyperleucocytose notée dans cette série pourrait être le fait d'une hyper production médullaire associée à une démargination permanente du pool marginal vers le pool circulant (Chies et al., 2001). Même si ce mécanisme n'est pas établi avec certitude, il est certain qu'il ne correspond pas à un phénomène non spécifique d'accompagnement d'une hyper production de la moelle érythroblastique. Cependant, l'absence de formule leucocytaire ne nous permet pas d'exclure les cas de fausses hyperleucocytoses secondaire à l'érythroblastose. En effet, dans les hémolyses aigues, la forte régénération médullaire est responsable d'une érythroblastose à l'origine d'une fausse hyperleucocytose, puisque les érythroblastes du fait de leur noyau sont comptés comme les leucocytes par les automates. Cette leucocytose non corrigée est souvent très importante chez les homozygotes. L'élévation de la CRP plaide en faveur d'une réaction inflammatoire susceptible d'augmenter le nombre de leucocytes puisqu'elle est corrélée de façon statistiquement significative à la leucocytose 
$(\mathrm{r}=0,72 ; \mathrm{p}<0,01)$.

Mounkaila et al. (2015) a rapporté chez les sujets SS un taux de moyen de plaquettes variant $511000 / \mathrm{mm}^{3}$. Cette tendance à la thrombocytose a été retrouvée par Nacoulma et al. (2006) avec une moyenne de $331000 / \mathrm{mm}^{3}$ chez le même type de patient. Nos résultats concordent avec ces chiffres, en effet nous avons observé une moyenne de $439000 \pm 139330 / \mathrm{mm}^{3}$. La thrombocytose observée pourrait s'expliquer par la survenue d'une asplénie fonctionnelle ou organique ou serait la conséquence d'une hyposplénie (Girot et al., 2004).

\section{Sur le plan Biochimique}

Dans cette série le taux moyen d'hémoglobine $\mathrm{S}$ était de 87,79 $\pm 8,2$ et est comparable avec les données de la littérature dont le taux variait entre 80 et $95 \%$ (Nagel et al., 2001). Le taux moyen de l'hémoglobine $F$ était de 9,5 $\pm 8,3 \%$. Des études menées au Sénégal ont montré chez les patients drépanocytaires homozygotes, un taux moyen d'hémoglobine $\mathrm{F}$ de $6,8 \%$ chez les enfants (Diagne et al., 2000) et de 8,2\% chez les adultes (Diop et al., 1999). L'hémoglobine F constitue le principal constituant hémoglobinique durant la période prénatale. Elle est synthétisée dès les premiers stades de la gestation pour attendre son taux maximal entre la huitième et la dixième semaine de la vie intra-utérine. Ce taux décroit dès la naissance pour ne rester qu'à l'état de trace (inférieur à $1 \%$ chez l'adulte normal) Labie 2000. Dans la drépanocytose, l'hémoglobine F interrompt le processus de polymérisation et diminue la rigidité cellulaire. Certaines thérapies de la drépanocytose visent à favoriser la dérépression de l'hémoglobine F. L'hémoglobine $F$ jouerait un rôle bénéfique dans la drépanocytose. Le taux en est cependant extrêmement variable s'échelonnant de 1 à $2 \%$ jusqu'à 25 à $30 \%$. Cette différence est particulièrement évidente chez les groupes homogènes d'origine ethnique différente (les sujets indiens et sénégalais expriment un taux d'hémoglobine F plus élevé que les autres sujets Africains), mais on la retrouve dans les familles de même origine ethnique et jusqu'entre les membres d'une même famille (Steinberg et al. 2003). Une corrélation relative et inconstante a été constatée entre le taux observé et la gravité de la maladie : un taux élevé laisse espérer une évolution moins sévère. Selon Powards (2005) il faut une élévation très sensible du taux d'hémoglobine $\mathrm{F}$ pour qu'il y ait réellement un effet bénéfique. Ainsi il a trouvé qu'un taux d'hémoglobine $\mathrm{F}$ supérieur à $10 \%$ réduisait la fréquence des complications alors qu'il fallait un taux d'hémoglobine $F$ supérieur à $20 \%$ pour réduire la fréquence des crises vaso-occlusives. Yan 2013 a constaté dans sa série qu'un taux d'hémoglobine $F$ supérieur à $10 \%$ était associé à un début tardif des symptômes et une présentation clinique peu sévère de la maladie caractérisée par peu d'hospitalisations et peu de transfusions. Dans cette série, le taux d'hémoglobine $\mathrm{F}$ était corrélée à la lithiase biliaire et au syndrome thoracique aigu $(\mathrm{r}=0,56 ; \mathrm{p}=0,003)$. Des résultats similaires ont été observés par Kueviakoe (2010) au Togo qui a noté que les complications anémiques et ischémiques étaient moins fréquentes chez les drépanocytaires homozygotes à taux d'hémoglobine $\mathrm{F} \geq 10 \%$.

La drépanocytose est une maladie inflammatoire dont l'un des marqueurs est la leucocytose. L'augmentation des leucocytes et leur activation sont des marqueurs importants de l'inflammation. Ce trouble parait donc être un signe spécifique de cette hémoglobinopathie puisque les patients présentant les signes d'une éventuelle infection ont été diminués, aussi l'augmentation de la CRP confirme la présence d'une réaction inflammatoire susceptible d'augmenter le nombre de globules blancs. Cette leucocytose est corrélée de façon statistiquement significative au taux de la CRP $(p=0.0036)$. Des résultats similaires ont été retrouvés par Gueye et al. (2014) qui avaient noté que le taux de CRP était plus élevé chez les drépanocytaires homozygotes en phase stationnaire que les témoins.

L'atteinte hépatique dans la drépanocytose n'est pas rare. Elle serait d'étiologie diverses : hépatite $C$, hépatite $B$, plus rarement, une hépatite liée aux crises 
vaso-occlusives spécifiques de la maladie du foie de surcharge martiale. L'hépatomégalie n'est pas constante. Dans cette série, la majorité des patients avait un bilan hépatique normal avec un taux d'ALAT et d'ASAT respectivement normal dans $73 \%$ et $82 \%$ des cas. Des résultats similaires ont été observés en Tunisie avec Hafsia et al. (2011). Le dysfonctionnement hépatique qu'il soit aigue ou chronique peut augmenter faussement la ferritinémie et expose à la surcharge en fer. La biopsie hépatique est la méthode de référence pour l'appréciation de la surcharge en fer (Karam et al. 2007). Vu les risques inhérents à la biopsie, d'autres techniques ont été développées (IRM, TDM). Certains auteurs Angelucci et al. (2000) ont objectivé une corrélation positive entre la ferritinémie et la valeur de fer hépatique par résonnance magnétique nucléaire.

\section{Conclusion}

Ces résultats sont semblables à ceux souvent décrits dans le syndrome drépanocytaire majeur. Faute de dépistage néonatal, le diagnostic est souvent posé en présence d'un signe d'appel. La meilleure connaissance des aspects biologiques devraient permettre de réduire la mortalité chez les homozygotes SS. Les paramètres hématologiques et biochimiques serviront de base de comparaison lors des crises et permettrons d'évaluer l'efficacité du traitement.

\section{CONFLIT D'INTERETS}

Les auteurs déclarent qu'ils n'ont pas de conflit d'intérêts.

\section{CONTRIBUTIONS DES AUTEURS}

$\mathrm{DD}, \mathrm{MD}, \mathrm{PMG}$ et $\mathrm{MS}$ ont contribué à la collecte des échantillons et à la rédaction du manuscrit; BFF, SMS, ont contribué au recrutement des patients; FD, AN, AS, FC, $\mathrm{AD}, \mathrm{ID}, \mathrm{SD}$ ont contribué à la relecture et à la correction du manuscrit.

\section{REFERENCES}

Angelucci E, Brittenham GM, Mclaren CE. 2000. Hepatic iron stores and plasma ferritin concentration in patients with sickle cell anemia and thalassemia major. New Engl Journal., 343(9): 32731.

Bardakjian J, Benkerrou M, Bernaudin F. 2000. Neonatal screening of sickle cell anemia in metropolitan France. Archives de Pediatrie, 7(12): 1261-1263. DOI : doi: 10.1136/jcp.2008.058867.

Chies JA, NardiI NB. 2001. Sickle cell disease: a chronic inflammatory condition. Medical Hypoth., 57(1): 4650.

Dahmani F, Saoud B, Jafaar K, Woumki A. 2016. Etude de l'hémogramme dans la drépanocytose homozygote à propos de 87 patients 2016. Pan African Medical Journal, 25: 240. doi:10.11604.

Diagne I, Ndiaye O, Moreira C, Signate -Sy H, Camara B. 2000. Les syndromes drépanocytaires majeurs à Dakar (Sénégal) Arch Pédiatr., 7(1): 16-24.

Diallo D. 2008. La drépanocytose en Afrique : problématique, stratégies pour une amélioration de la survie et de la qualité de vie du drépanocytaire, Bull. Acad. Natle Méd., 192(7): 1361-1373.

Diop S, Thiam D, Cisse M, Touré AO, Fall K, Diakhaté L. 1999. New result in clinical severity of homozygous sickle cell anemia in Dakar, Sénégal. Hematol cell Ther., 41(1): 217-221.

Doumbia A. 2009. Aspects épidemiocliniques des enfants drépanocytaires suivis en Pédiatrie de 2005 - 2008. Thèse de médecine, Université du Mali, Mali, p.96

Dokékias A, Nzingoula S. 2001 Profil du sujet drépanocytaire après l'âge de 30 ans. Médecine d'Afrique Noire, 48(10): 411418.

Gueye PM, Gueye-Tall F, Seck M, Kane MO, Diallo RNd, Diatta M. 2014. Aggravation de l'anémie et polymorphisme de l'haptoglobine au cours de la drépanocytose au Sénégal. International Journal of Biological and Chemical Sciences, 8(3): 975-982.

Girot R, Bégué P, Binet JL, Vacheron A, Queneau P, Sraer JD. 2004. La drépanocytose chez l'enfant. Bull Acad Nat de Méd., 188(3): 491-506. 
Godeau B. 2001. Prise en Charge des Syndromes Drépanocytaires Majeurs ( $4^{\text {ème }}$ édn, tome 1). Médecine-science Flammarion : Paris.

Hafsia R, Belakhal F, Ben Sallah N, Gouider E, Borgi W. 2011. Evaluation de la surcharge en fer au cours de la drépanocytose: à propos de 94 cas. $L a$ Tunisie Médicale, 89(6): 548-552.

Harrak A, Ouahmane S, Benhsaien I, Maani K, Hachim J, Hadjkhalifa H. 2010. Drépanocytose chez l'enfant à l'unité d'hémato-oncologie pédiatrique. Archives de Pédiatrie, 17(5): 157-163.

Karam LB, Disco D, Jackson SM, Lewin D, McKie V, Baker RD, Baker SS, Laver JH, Nietert PJ, Abboud MR. 2007. Liver biopsy results in patients with sickle cell disease on chronic transfusions: Poor correlation with ferritin levels. Pediatr Blood Cancer, 24(5): 520-525.

Kueviakoe MI, N'zouonta V, Agbetiafa K, Padaro E, Vovor A, Segbena AY. 2010. Influence du taux d'hémoglobine F sur le profil évolutif de la drépanocytose homozygote au Togo. Journal de Recherche Scientifique de l'Université du Togo, 12(2): 243-248.

Labie D. 2000. Pourquoi l'hémoglobine F estelle élevée dans les thalassémies, l'hétérozygotie E/béta-thalassémie comme modèle. Revue d'Hématologie, 6(5) : 87-93.

Mabiala BJR, Nkanza KSAT, Ganza ZPS, Nzingoula S, Senga P. 2005. Causes d'hospitalisation des enfants drépanocytaires: influence de l'âge (CHU de Brazzaville, Congo). Bull. Soc. Pathol. Exot., 98(5): 392-393.

Marie Redelsperger M, BardakdjlanMichau J, Neonat OMG, Girot R. 2003. Diagnostic biologique des syndromes drépanocytaires, In La Drépanocytose, Girot R, Bégué P, Galacteros F (eds). John Libbey Eurotext: Paris; 1329.

Monkaila B, Oumarou HK, Garba M, Maiga A, Akpona SA, Sanogo I. 2015. Hémolyse chronique des sujets drépanocytaires SS et SC en phase stationnaire: étude comparative au centre national de référence de la drépanocytose à Niamey. Revue Cames Sante, 3(1) : 25-29.

Nacoulma E, Sakande J, Kafando E, Kpowbié ED, Guissou IP 20006. Profil hématologique et biochimique des drépanocytaires SS et SC en phase stationnaire au Centre Hospitalier National Yalgado Ouedraogo de Ouagadougou. Mali Medical., 21(1): 811.

Nagel RL, Steinberg MH. 2001. Hémoglobine SS Disease and Hemogline SC Disorders; Disorders of Hemoglobine. Cambridge University Press: Cambribge; 756-785.

Powards DR, Chan LS, Hiti A. 2005. Outcome of sickle cell anemia: a 4decade observational study 1056 patients. Medecine (Baltimore), 84(3): 363-376.

Serjeant GR. 2013. The natural history of sickle cell disease. Cold Spring Harbor Perspectives in Medicine, 1(3): 153-157. DOI: 10.1101/cshperspect.a011783.

Shongo M, Mukuku O, Mutombo A, Loubala T. 2015. Profil hématologique et nutritionnel du drépanocytaire homozygote SS âgés de 6 à 59 à Lubumbashi, République Démocratique du Congo. Pan African Medical Journal, 21: 276. doi:10.11604

Steinberg MH, Barton F, Castro O. 2003. Effect of hydroxyurea on mortality and morbidity in adult sickle cell anemia: risks and benefits up to 9 years of treatment. JAMA, 289(13): 1645 - 1651 DOI.101001

Than THC, Mekinian A, Godineau M, Rose C 2008. Rétinopathie chez les adultes de la Région Nord-Pas-de Calais. Journal Français d'Ophtalmologie, 31(10): 987992.

Yan CME 2013. Persistance de l'hémoglobine foetale chez les enfants drépanocytaires homozygotes ages de 2 à 18 ans suivis au CME/FCB. Journal of Medecine and Health Sciences, 12(2): 88-93. 